\section{PONTOS DE TRANSIÇÃO DA FREQUÊNCIA CARDÍACA NA MARCHA ATLÉTICA}

\author{
TRANSITION POINTS OF HEART RATEAT RACE WALKING
}

PUNTOS DE TRANSICIÓN DE LA FRECUENCIA CARDÍACA EN LA MARCHA ATLÉTICA

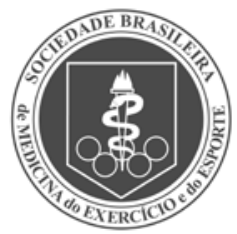

Artigo Original

Original ARticle

Artículo Original
Danilo Leonel Alves ${ }^{1}$

(Profissional de Educação Física)

Ramon $\mathrm{Cruz}^{2}$

(Profissional de Educação Física)

Francisco de Assis Manoel ${ }^{3}$

(Profissional de Educação Física)

Pablo Ramon Domingos ${ }^{4}$

(Profissional de Educação Física)

Jefferson Verbena de Freitas ${ }^{4}$

(Profissional de Educação Física)

Raul Osiecki

(Profissional de Educação Física)

Fernando Roberto de Oliveira ${ }^{5}$

(Profissional de Educação Física)

Jorge Roberto Perrout de Lima ${ }^{4}$

(Profissional de Educação Física)

1. Universidade Federal do Paraná (UFPR), Curitiba, PR, Brasil.

2. Universidade de São Paulo (USP),

São Paulo, SP, Brasil.

3. Universidade Estadual de

Maringá (UEM), Maringá, PR, Brasil.

4. Universidade Federal de Juiz de Fora (UFJF), Juiz de Fora, MG, Brasil. 5. Universidade Federal de Lavras (UFLA) Lavras, MG, Brasil.

\section{Correspondência:}

Rua Coração de Maria, 92, BR

116, Km 95, Jardim Botânico,

Curitiba, PR, Brasil. 80215-370.

daniloleoneledufisica@gmail.com

\section{RESUMO}

Introdução: A frequência cardíaca fornece informações úteis para os treinamentos de marcha atlética. Objetivo: O objetivo do estudo foi analisar o comportamento da frequência cardíaca (FC) e seus pontos de inflexão (PIFC) e deflexão (PDFC) em teste progressivo de marcha atlética (TPMA) antes e depois de 20 sessões de treinamento. Métodos: Participaram 13 jovens atletas $(12,46 \pm 1,61$ anos, 44,29 $\pm 10,25 \mathrm{~kg}, 157,93 \pm 12,03 \mathrm{~cm}, 24,39 \pm 7,60$ $\%$ G). OTPMA foi realizado em uma pista oficial de atletismo, antes e depois do treinamento. Os dados de FC e carga foram plotados a cada minuto para identificação dos PIFC e PDFC. Resultados: A FC apresentou comportamento sigmoide, com identificação dos pontos de transição (PT), sendo no pré-treinamento: a) oito sujeitos $\operatorname{PIFC}\left(5,31 \mathrm{~km} \cdot \mathrm{h}^{-1} ; 125 \mathrm{bpm}\right)$ e PDFC $\left.\left(7,63 \mathrm{~km} \cdot \mathrm{h}^{-1} ; 169 \mathrm{bpm}\right) ; \mathrm{b}\right)$ um sujeito somente PIFC $\left(7,00 \mathrm{~km} \cdot \mathrm{h}^{-1} ; 149 \mathrm{bpm}\right)$; c) um sujeito somente PDFC $\left(8,00 \mathrm{~km} \cdot \mathrm{h}^{-1} ; 170 \mathrm{bpm}\right)$; $)$ três sujeitos sem detecção de PT e no pós-treinamento: a) em 12 sujeitos PIFC (5,46 km $\left.\mathrm{h}^{-1} ; 125 \mathrm{bpm}\right)$ e PDFC (7,75 km.h $\left.\mathrm{h}^{-1} ; 168 \mathrm{bpm}\right)$; b) um sujeito somente PDFC (7,50 $\left.\mathrm{km} \cdot \mathrm{h}^{-1} ; 184 \mathrm{bpm}\right)$. O PIFC foi encontrado em carga significativamente inferior ao PDFC no pré $(p<0,001)$ e no pós-treinamento $(p<0,001)$. Quando comparamos o PIFC e o PDFC pré e pós, não encontramos diferença significativa, seja em relação à carga ( $p=0,87$ e $p=0,61)$ ou $F C(p=0,60$ e $p=0,99)$. Conclusão: Conclui-se que a FC tem relação curvilínea com a carga, sendo possível detectar os seus pontos de transição em TPMA.

Descritores: consumo de oxigênio; frequência cardíaca; educação física e treinamento; atletismo.

\section{ABSTRACT}

Introduction: Heart rate provides useful information for race walking training. Objective: The objective of study was to analyze the behavior of heart rate (HR) and inflection points (HRIP) and deflection points (HRDP) in a progressive test of race walking (PTRW) before and after 20 training sessions. Methods: Participants were 13 young athletes $(12.46 \pm 1.61$ years, 44.29 $\pm 10.25 \mathrm{~kg}, 157.93 \pm 12.03 \mathrm{~cm}, 24.39 \pm 7.60 \mathrm{~F} \%)$. The PTRW was held at an official athletics track before and after training. The HR data and load were plotted every minute to identify the HRIP and HRDP. Results: The HR showed sigmoid behavior with the identification of transition points (TP), being in the pre-training: a) eight subjects HRIP (5.31 km. $\left.h^{-1} ; 125 \mathrm{bpm}\right)$ and $\operatorname{HRDP}\left(7.63 \mathrm{~km} \cdot \mathrm{h}^{-1} ; 169 \mathrm{bpm}\right) ;$ b) one subject only HRIP $\left.\left(7.00 \mathrm{~km} \cdot \mathrm{h}^{-1} ; 149 \mathrm{bpm}\right) ; \mathrm{c}\right)$ one subject only HRDP $\left(8,00 \mathrm{~km} \cdot \mathrm{h}^{-1} ; 170\right.$ bpm); d) three subjects had no TP observed, and post-training: a) 12 subjects HRIP (5.46 km. $h^{-1} ; 125$ bpm) and HRDP (7.75 $\left.\mathrm{km} \cdot \mathrm{h}^{-1} ; 168 \mathrm{bpm}\right)$; b) one subject HRDP $\left(7.50 \mathrm{~km} \cdot \mathrm{h}^{-1} ; 184 \mathrm{bpm}\right)$. The HRIP was found to be significantly lower than the HRDP in pre-training $(p<0.001)$ and post-training $(p<0.001)$. When we compared HRIP and HRDP in the pre-and post-training we found no significant difference either in relation to the load ( $p=0.87$ and $p=0.61)$ or HR ( $p=0.60$ and $p=0.99$ ). Conclusion: It is concluded that the HR has curvilinear relation with the load, and that it is possible to detect its transition points during PTRW.

Keywords: oxygen consumption; heart rate; physical education and training; track and field.

\section{RESUMEN}

Introducción: La frecuencia cardiaca proporciona información útil para los entrenamientos de marcha atlética. Objetivo: El objetivo del estudio fue analizar el comportamiento de la frecuencia cardiaca (FC) y sus puntos de inflexión (PIFC) y de deflexión (PDFC) en prueba progresiva de marcha atlética (PPMA) antes y después de 20 sesiones de entrenamiento.

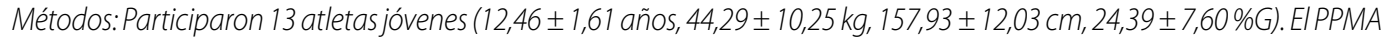
fue realizado en una pista oficial de atletismo antes y después del entrenamiento. Los datos de FC y carga fueron trazados cada minuto para identificación de PIFCy PDFC. Resultados: La FC presentó comportamiento sigmoideo, con identificación de los puntos de transición (PT), siendo en el pre-entrenamiento: a) ocho sujetos PIFC (5,31 km.h $\left.h^{-1}, 125 \mathrm{lpm}\right)$ y PDFC (7,63 $\left.\left.\mathrm{km} \cdot \mathrm{h}^{-1} ; 169 \mathrm{lpm}\right) ; \mathrm{b}\right)$ un sujeto solamente PIFC $\left.\left(7,00 \mathrm{~km} \cdot \mathrm{h}^{-1} ; 149 \mathrm{lpm}\right) ; \mathrm{c}\right)$ un sujeto solamente PDFC $\left.\left(8,00 \mathrm{~km} \cdot \mathrm{h}^{-1}, 170 \mathrm{lpm}\right) ; \mathrm{d}\right)$ tres sujetos sin detección de PTy post-entrenamiento: a) 12 sujetos PIFC $\left(5,46 \mathrm{~km} \cdot \mathrm{h}^{-1}, 125 \mathrm{lpm}\right)$ y PDFC $\left(7,75 \mathrm{Km} \cdot \mathrm{h}^{-1} ; 168 \mathrm{lpm}\right)$; b) un sujeto solamente PDFC (7,50 km. $\left.h^{-1}, 184 \mathrm{lpm}\right)$. El PIFC fue encontrado en carga significativamente inferior que el PDFC en el pre $(p<0,001)$ y en el post-entrenamiento $(p<0,001)$. Al comparar el PIFC y el PDFC prey post-entrenamiento, no se encontró ninguna diferencia significativa, sea en relación a la carga $(p=0,87$ y $p=0,61)$ o FC $(p=0,60 ; p=0,99)$. Conclusión: Se concluye que la FC tiene relación curvilínea con la carga, siendo posible detectar sus puntos de transición en PPMA.

Descriptores: consumo de oxígeno; frecuencia cardíaca; educación y entrenamiento físico; atletismo. 


\section{INTRODUÇÃO}

A marcha atlética é uma prova do atletismo que exige do atleta grande domínio técnico, resistência aeróbia, flexibilidade e coordenação, e o treinamento desses aspectos é fundamental para o aumento do desempenho'. Para a avaliação e o controle dos treinamentos, a frequência cardíaca ( $F C$ ) tem sido bastante estudada durante diferentes tipos e condições associadas ao exercício, por apresentar grande facilidade de mensuração, além de sua utilidade para a avaliação aeróbia e estimativa do gasto calórico². Durante décadas, a abordagem clássica de relação linear FC-Carga ${ }^{3-5}$ e com o consumo de oxigênio $\left(\mathrm{VO}_{2}\right)$ foi a que mais se difundiu entre os pesquisadores e, consequentemente, a mais relatada nos trabalhos apresentados pela literatura no estudo do comportamento da FC em teste progressivo máximo (TPM). Diferentemente do acima proposto, Conconi et al. ${ }^{6}$ e Lima ${ }^{7}$, sustentam um comportamento não linear da FC-Carga. Com esta abordagem, há possibilidade de identificação do fenômeno fisiológico denominado "Pontos de Transição (PT) da FC". Especificamente, o PT seria o momento que, com o aumento da intensidade de trabalho, haveria uma ruptura na linearidade da $F^{6,8,89}$. Assim, em análises de $F C$ com cargas progressivas de trabalho são possíveis identificar dois pontos de mudança de direção, que correspondem aos PT's metabólicos durante o esforço ${ }^{10}$.

O primeiro PT da quebra da linearidade da FC é identificado em baixas intensidades durante a fase côncava da $\mathrm{FC}^{11,12}$. Observando o comportamento das distintas porções da curva FC x intensidade de esforço, Lima ${ }^{7}$ apresentou a partir de um ajuste sigmoide (próximo a um "S"), um PT denominado de ponto de inflexão da frequência cardíaca (PIFC), sugerindo que em baixas intensidades ocorre a aceleração da FC devido à retirada vagal e aumento da atividade simpática na modulação autonômica, sendo este evento observado em intensidades similares ao limiar de lactato ${ }^{13,14}$.

Para a identificação do PIFC, são necessárias baixas cargas iniciais, que permitem uma análise mais completa do comportamento da variável com o incremento da intensidade de trabalho. Alguns autores começaram a utilizar este evento como descritor de primeiro limiar de transição fisiológica $\left(\mathrm{LT}_{1}\right)$, o descrevendo como o ponto em que ocorre a primeira quebra da linearidade da $F C^{12,15}$.

O segundo PT da FC, denominado de ponto de deflexão da frequência cardíaca (PDFC), consiste na fase convexa da FC, representando um ponto de quebra da linearidade da $\mathrm{FC}$ em cargas próximas à velocidade máxima, estando associado ao segundo limiar de lactato $\left(L L_{2}\right)^{6,16}$. Observando a curva da FC em teste com incrementos de velocidade a cada 200 metros de corrida percorridos, Conconi et al., ${ }^{6,17}$ e Lima foram os primeiros a descrever o PDFC na literatura. Estes autores identificaram o PDFC em cargas similares ao $L_{2}$. Desde então, o PDFC vem sendo utilizado como indicador do $L L_{2}$ e parece estar bem elucidado na literatura 16,18-20. Diversos trabalhos têm abordado a questão da existência dos PT's, como no ciclismo, marcha atlética, natação, remo e atletismo $6,9,11,17,20-22$ e sua aplicabilidade no universo do treinamento físico, porém os trabalhos concentraram-se em investigar o comportamento da FC em protocolos que apresentavam cargas de trabalhos iniciais com intensidades médias e/ou incrementos de carga acima de $0,5 \mathrm{~km} \cdot \mathrm{h}^{-123}$, o que pode dificultar a elaboração de uma curva mais precisa e, consequentemente, a identificação dos PT's. Esse fator pode ser responsável pela controvérsia na existência ou não dos PT's ${ }^{11}$. Além disso, com raras exceções ${ }^{7}$, são estudadas as partes superiores da curva, sendo descritos, basicamente, os PT's obtidos em cargas próximas ao $L_{2}$. Cambri et al. ${ }^{11}$ verificaram que em teste progressivo máximo em esteira ergométrica é possível identificar os dois PT's, embora nem sempre em conjunto.
Para a marcha atlética, treinadores e atletas precisam de informações úteis nas tomadas de decisão na produção e execução do treinamento. De nosso conhecimento, apenas um estudo ${ }^{24}$ investigou os pontos de transição da frequência cardíaca em teste progressivo de marcha atlética (TPMA) no qual foi identificado o PDFC utilizando os métodos de inspeção visual ${ }^{17}$ e Dmáx ${ }^{25}$. Porém, não são encontrados estudos com a identificação do PIFC e PDFC em TPMA utilizando o modelo matemático de Cambri et al.1" e comparando também, os efeitos do treinamento da prova.

Diante deste contexto, o objetivo do presente estudo foi analisar o comportamento da FC e identificar os PT's (PIFC e PDFC) em teste progressivo de marcha atlética em pista. Em um segundo momento comparar estas variáveis após o treinamento específico da prova. Hipotetizamos que é possível detectar os pontos de transição da frequência cardíaca no TPMA, e que o treinamento pode ocasionar alterações no comportamento da FC.

\section{MATERIAIS E MÉTODOS}

A amostra foi do tipo intencional, com a participação voluntária de treze jovens atletas, com idade entre 10 e 14 anos, sendo três do sexo masculino e 10 do sexo feminino $(12,46 \pm 1,61$ anos, $44,29 \pm 10,25 \mathrm{Kg}$, $157,93 \pm 12,03 \mathrm{~cm}, 24,39 \pm 7,60 \% \mathrm{G})$ sem histórico de doenças graves, não treinados especificamente para a prova de marcha atlética e com uma frequência de treinamento de cinco sessões semanais. Todos os sujeitos e seus responsáveis legais foram devidamente informados sobre os procedimentos dos testes e assinaram respectivamente, o Termo de Assentimento e de Consentimento Livre e Esclarecido, aprovado pelo comitê de ética da Universidade Federal de Lavras (974.644 / 2015).

\section{Desenho experimental}

Foram feitas indicações prévias para os sujeitos quanto aos hábitos adotados no período anterior ao teste, como não ingerir café nas 24 horas antecedentes, além de não consumir alimentos por duas horas antes da sua realização, bem como não fazer exercícios físicos extenuantes no dia precedente.

As avaliações antropométricas foram mensuradas antes do início dos testes, sendo: estatura (estadiômetro Asimed ${ }^{\circledR}$, Espanha), massa corporal (balança portátil Britânia ${ }^{\circledR}$, Brasil) e dobras cutâneas das regiões do tríceps e subescapular (adipômetro Sanny ${ }^{\circledR}$, Brasil), conforme a padronização proposta por McArdle et al. ${ }^{26}$ para detecção da densidade corporal, seguido da fórmula de Siri para encontro da \% de gordura corporal.

O teste progressivo de marcha atlética (TPMA) foi realizado em uma pista oficial de atletismo com superfície de saibro de acordo com o protocolo apresentando por Frainer et al. ${ }^{24}$. O TPMA foi precedido de uma mobilização geral, de baixa intensidade, para aquecimento prévio à atividade. O TPMA iniciou com uma velocidade de $4 \mathrm{~km} \cdot \mathrm{h}^{-1} \mathrm{com}$ incrementos de $0,5 \mathrm{~km} \cdot \mathrm{h}^{-1}$ a cada minuto. A velocidade durante o teste foi controlada pelos sinais sonoros a partir de software específico, os avaliados deveriam cruzar a linha dos cones, que foram distribuídos na pista a cada 50 metros, com pelo menos um dos pés simultaneamente ao sinal sonoro (BIP), o ritmo deveria ser respeitado rigorosamente. $O$ intervalo entre os BIPs em cada velocidade diminuia, desta forma o avaliado foi aumentando progressivamente a sua velocidade de marcha. Os testes foram mantidos até exaustão voluntária e os participantes foram encorajados verbalmente a se manterem em esforço pelo maior tempo possível. O teste foi encerrado, quando o participante ou quando o avaliador identificar que o mesmo não conseguiu por duas vezes consecutivas ou três vezes alternadamente, ultrapassar com um dos pés a linha do cone.

O pico de velocidade foi considerado a máxima velocidade de atingida durante o TPMA; caso o participante não concluísse o último estágio 
iniciado, o pico de velocidade foi calculada com base no tempo parcial permanecido no último estágio atingido, a partir da equação proposta por Kuipers. A FC foi registrada ao final de cada estágio, utilizando o cardiofrequêncímetro Polar ${ }^{\circledR}$ (S810i, Finlândia).

Os dados da FC e da carga foram plotados a cada minuto para identificação dos PIFC e PDFC através do modelo matemático de Cambri et al. ${ }^{11}$. Neste modelo, todos os valores FC - cargas são ajustadas por uma função polinomial de terceiro grau e por uma equação linear de primeiro grau derivadas dos dados de cada um dos sujeitos. Posteriormente, foi calculada a diferença dos valores de FC obtidos pelas respectivas equações, e projetada uma curva com estes valores. O PIFC e o PDFC foram identificados respectivamente, nos menores e maiores valores antes da mudança na direção da curva.

Após o primeiro TPMA, foram realizadas 20 sessões com duração entre 30 e 60 minutos de treinamento geral de marcha atlética, com objetivo de aprimorar o condicionamento físico para a prova de marcha atlética. Este treinamento foi composto por diversas atividades, tais como exercícios coordenativos específicos (educativos), tiro máximo em pista, fartlek, marcha com finalidades aeróbias e atividades recreacionais. Logo após o término das 20 sessões de treinamento, foi realizado mais um TPMA.

\section{Análise estatística}

Foi utilizada a estatística descritiva para a apresentação dos resultados (média \pm desvio padrão). A normalidade dos dados foi verificada pelo teste de Shapiro-Wilk. Para as comparações das variáveis carga $\left(\mathrm{km} \cdot \mathrm{h}^{-1}\right)$ e FC (bpm) antes e depois do treinamento foi utilizado o teste t de Student de amostras pareadas e a correlação r de Pearson. Todas as análises foram feitas no software SPSS (v.20, SPSS Inc., Chicago, IL, USA), sendo adotado o nível de significância de 5,0 \% ( $p<0,05)$. Para a análise da variabilidade da FC e seus respectivos PT's utilizamos o programa Graphpad Prism, versão 5.00 (2007 - Graphpad Software Incorporated ${ }^{\circledR}$ ).

\section{RESULTADOS}

Neste trabalho foi estudado o comportamento da FC em TPMA em pista de saibro, com cargas de trabalho iniciais de baixa intensidade e pequenos incrementos de carga, objetivando investigar a existência de PT na prova estudada. Para ilustrar esta relação curvilínea entre FC e a carga de trabalho, utilizamos o gráfico de um avaliado nos testes pré e pós sessões de treinamento (Figura 1):

A análise da curva da FC demonstrou um comportamento curvilíneo, com detecção de PT em 10 sujeitos no pré-treinamento e em 13 sujeitos no pós- treinamento. A Tabela 1 apresenta os valores descritivos (Média e DP) dos pontos de transição da FC no pré-treinamento. Em oito atletas a carga e a FC do PDFC foi, significativamente, maior do que o PIFC, permitindo assim, a associar os PT's aos limiares de lactato.
Após as 20 sessões de treinamento, foi possível identificar os PIFC e PDFC em 12 sujeitos, sendo que, a carga e a FC do PDFC foi maior, significativamente, que o PIFC. A Tabela 2 apresenta os valores descritivos destas variáveis no pós-treinamento.

O PIFC foi encontrado em cargas significativamente inferiores ao PDFC seja no pré ou pós-treinamento (tanto para a carga quanto para a frequência cardíaca).

Quando comparamos os PT's encontrados pré e pós-treinamento, observou-se que não há diferença significante entre as variáveis PIFC pré x PIFC pós e PDFC pré x PDFC pós, seja para a carga ou para a frequência cardíaca (Tabela 3).

\section{DISCUSSÃO}

Este trabalho teve como objetivo analisar o comportamento da FCe identificar os PT's (PIFC e PDFC) em teste progressivo de marcha atlética em pista e comparar estas variáveis após o treinamento específico da prova em jovens atletas. Os principais achados foram a possibilidade de se detectar os pontos de transição em TPMA e que 20 sessões de treinamento não alteram a carga e na FC em que os PT's são atingidos. Este último dado encontrado contraria as expectativas após as sessões

Tabela 1. PIFC e PDFC pré-treinamento.

\begin{tabular}{|c|c|c|c|c|c|}
\hline \multicolumn{6}{|c|}{ Pré-treinamento } \\
\hline & \multicolumn{2}{|c|}{ PIFC e PDFC } & \multirow{2}{*}{$\begin{array}{c}\text { Somente } \\
\text { PIFC }\end{array}$} & \multirow{2}{*}{$\begin{array}{l}\text { Somente } \\
\text { PDFC }\end{array}$} & \multirow{2}{*}{$\begin{array}{c}\text { PT's não } \\
\text { encontrados }\end{array}$} \\
\hline & PIFC & PDFC & & & \\
\hline $\mathrm{N}$ & \multicolumn{2}{|c|}{8} & 1 & 1 & 3 \\
\hline Carga $\left(\mathrm{km} \cdot \mathrm{h}^{-1}\right)$ & $5,31 \pm 0,80$ & $7,63^{*} \pm 1,03$ & 7,00 & 8,00 & \\
\hline $\mathrm{FC}(\mathrm{bpm})$ & $125 \pm 25$ & $169^{*} \pm 36$ & 149 & 170 & \\
\hline
\end{tabular}

Tabela 2. PIFC e PDFC pós-treinamento.

\begin{tabular}{|c|c|c|c|}
\hline \multicolumn{4}{|c|}{ Pós-treinamento } \\
\hline & \multicolumn{2}{|c|}{ PIFC e PDFC } & \multirow{2}{*}{ Somente PDFC } \\
\hline & PIFC & PDFC & \\
\hline $\mathrm{N}$ & \multicolumn{2}{|c|}{12} & 1 \\
\hline Carga $\left(\mathrm{km} \cdot \mathrm{h}^{-1}\right)$ & $5,46 \pm 0,45$ & $7,75^{*} \pm 0,81$ & 7,5 \\
\hline $\mathrm{FC}(\mathrm{bpm})$ & $125 \pm 14$ & $168^{*} \pm 12$ & 184 \\
\hline
\end{tabular}

Tabela 3. Comparação entre os PT's pré e pós-treinamento de todos os sujeitos.

\begin{tabular}{c|c}
\hline & Valor de $\mathbf{p}$ \\
\hline PIFC Pré x PIFC Pós - Carga $\left(\mathrm{km} \cdot \mathrm{h}^{-1}\right)$ & 0,87 \\
\hline PIFC Pré x PIFC Pós - FC $(\mathrm{bpm})$ & 0,60 \\
\hline PDFC Pré x PDFC Pós - Carga $\left(\mathrm{km} \cdot \mathrm{h}^{-1}\right)$ & 0,61 \\
\hline PDFC Pré x PDFC Pós - FC $(\mathrm{bpm})$ & 0,99 \\
\hline
\end{tabular}
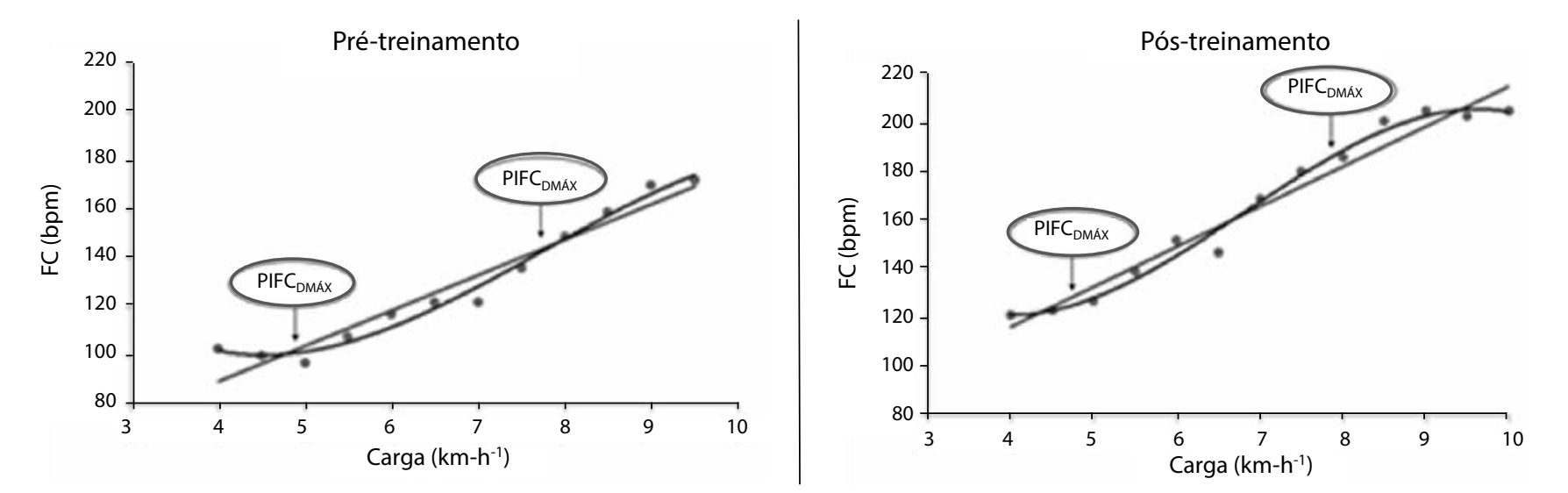

Figura 1. Demonstração dos PT's gerados pela relação sigmoide entre carga x FC utilizando o método Dmáx. Dados reais de um sujeito pré e pós-treinamento. 
de treinamento, podendo ser consequência do pequeno tempo de treinamento (20 sessões) e pela grande aptidão que se encontravam os avaliados (treinados em outras provas do atletismo), o que não geraria alterações consideráveis no PT's em curto prazo. Portanto, para este número de sessões, não houve alterações significativas na detecção de PT's após o treinamento de marcha atlética.

Os resultados do estudo demonstraram que é possível identificar os PT's dos sujeitos em TPMA, porém, em três deles os pontos não foram encontrados em conjunto (PIFC e PDFC). Para o PIFC, apesar dos mecanismos fisiológicos não sejam completamente compreendidos, Alonso et al. ${ }^{5}$ sugerem que no início do exercício progressivo máximo ocorre retirada parassimpática, e nos estágios subsequentes do teste ocorre incremento da atividade simpática. Esses ajustes permitem a identificação do PIFC, com baixas cargas iniciais, pequenos incrementos de intensidade e estágios de curta duração.

Para o PDFC, as influências parecem estar ligadas a função do miocárdio, a atividade do sistema neural e o nível de catecolaminas e de potássio $(K+)^{20,27}$. Os nervos simpáticos cardíacos estimulam a liberação das catecolaminas adrenalina e noradrenalina, aumento consequentemente, a contratilidade miocárdica. Assim, o débito cardíaco permanece elevado em altas intensidades sem que ocorram grandes aumentos na FC $^{28,29}$. O aumento da concentração plasmática de potássio $(K+)$ também faz com que aumente o volume de ejeção, provocando um aumento menos pronunciado da $\mathrm{FC}^{20}$.

Adicionalmente, Hofmann et al. ${ }^{30}$ apresenta a hipótese de que a origem fisiológica do PDFC seja a sensibilidade dos ß1-adrenoreceptores. Os adrenoreceptores causam uma resposta simpática, com aumento da FC e redimensionamento do fluxo sanguíneo de órgãos não essenciais durante o exercício para o músculo esquelético. Desta forma, em cargas acima do segundo limiar, quando há aumento das catecolaminas, os receptores estão saturados e não ocorrem grandes aumentos da FC, preservando assim a função ventricular esquerda ${ }^{20,30}$.
Porém, os exatos mecanismos fisiológicos sobre os PT's ainda não estão completamente esclarecidos.

A identificação de PT da FC utilizando o método Dmáx adaptado por Cambri et al. ${ }^{11}$ parece ser objetiva, prática e de baixo custo operacional. No presente estudo foi possível identificar os pontos de transição em teste progressivo de marcha atlética (TPMA) em pista e comparar estes após sessões de treinamento. Porém, nossos resultados limitam-se por não serem comparados com medidas diretas de lactato sanguíneo e/ou ventilação durante o teste progressivo máximo empregado e não ter verificado os efeitos do treinamento em longo prazo. Os PT a partir de curvas da FC podem ser úteis na prescrição e acompanhamento de treinamento, mas sua aplicação pode ser limitada, pois nem todos os sujeitos apresentam esses fenômenos em conjunto. Devido ao aumento das identificações de PT em diferentes situações práticas, sugerimos que mais estudos sejam realizados com estas mesmas características, porém, recomendamos agora, a verificação dos efeitos do treinamento em longo prazo.

Apesar das limitações apresentadas, a validade ecológica do nosso estudo é bastante atraente, pois foram avaliados jovens atletas de atletismo, com testes de campo específicos da prova. Preconiza-se que para as tomadas de decisão no treinamento, testes como o utilizado por este estudo sejam considerados.

\section{CONCLUSÃO}

A frequência cardíaca em teste progressivo máximo de marcha atlética em pista tem relação curvilínea com a carga e é possível detectar os seus pontos de transição, denominados PIFC e PDFC. O PIFC foi encontrado em cargas relativamente menores que o PDFC. Vinte sessões de treinamento não interferem nos valores de carga e de FC dos PIFC e PDFC.

Todos os autores declararam não haver qualquer potencial conflito de interesses referente a este artigo.

CONTRIBUIÇÕES DOS AUTORES: Cada autor contribuiu individual e significativamente para o desenvolvimento do manuscrito. DLA (0000-0001-7583-0322)* e RC $(0000-0002-1308-5993)^{*}$ foram os principais contribuintes na coleta de dados e redação do manuscrito. FAM (0000-0003-1841-5637)*, PRD (0000-0003-3928-922X)* e JVF (0000-0002-9897-3729)* auxiliaram na coleta de dados, análise dos resultados e revisão do manuscrito. RO (0000-0002-3573-9468)*, JRPL (0000-0003-0073-8673)* e FRO (0000-0003-2811-8433)* realizaram a revisão do manuscrito e contribuíram com o conceito intelectual do estudo. *ORCID (Open Researcher and Contributor ID).

\section{REFERÊNCIAS}

1. Oliveira TI. Aprendendo a Marcha Atlética. Projeto de Intervenção Pedagógica na escola, apresentado como atividade do Plano de Formação Continuada do Programa de Desenvolvimento Educacional da Secretaria de Estado da Educação do Paraná. São José dos Pinhais, PR: Universidade Federal do Paraná; 2008.

2. Almeida MB, Araújo CGS. Efeitos do treinamento aeróbico sobre a freqüência cardíaca. Rev Bras Med Esporte. 2003;9(2):104-12

3. Wahlund H. Determination of the physical working capacity: a physiological and clinical study with special reference to standardization of cardio-pulmonary functional tests. Act Med Scand Suppl. 1948;132(215):1-78

4. Astrand PO, Ryhming I. A nomogram for calculation of aerobic capacity (physical fitness) from pulse rate during sub-maximal work. J Appl Physiol. 1954;7(2):218-21.

5. Alonso DO, Forjaz CLD, Rezende LO, Braga AMF, Barretto ACP, Negrão CE, et al. Comportamento da frequência cardíaca e da sua variabilidade durante as diferentes fases do exercício físico progressivo máximo. Arq Bras Cardiol. 1998;71(6):787-92.

6. Conconi F, Ferrari M, Ziglio PG, Droghetti P, Codeca L. Determination of the anaerobic threshold by a noninvasive field test in runners. J Appl Physiol Respir Environ Exerc Physiol. 1982;52(4):869-73.

7. Lima JRP. Frequência cardíaca em cargas crescentes de trabalho: ajuste sigmoide, ponto de inflexão e limiar de variabilidade da frequência cardíaca [tese]. São Paulo: Universidade de São Paulo, Escola de Educação Física e Esporte; 1997.

8. Droghetti P, Borsetto C, Casoni I, Cellini M, Ferrari M, Paolini AR, et al. Noninvasive determination of the anaerobic threshold in canoeing, cross-country skiing, cycling, roller, and ice-skating, rowing, and walking. Eur J Appl Physiol Occup Physiol. 1985:53(4):299-303.

9. Cellini M, Vitiello P, Nagliati A, Ziglio PG, Martinelli S, Ballarin E, et al. Noninvasive determination of the anaerobic threshold in swimming. Int J Sports Med. 1986;7(6):347-51.

10. Cambri LT. Modulação autonômica cardíaca e controle metabólico em diabetes tipo 2 em repouso e exercício [dissertação]. Florinópolis, SC: Universidade do Estado de Santa Catarina; 2007.

11. Cambri LT, Foza V, Nakamura FY, De-Oliveira FR. Frequência cardíaca e a identificação dos pontos de transição metabólica em esteira rolante. Rev Educ Fis. 2006;17(2):131-7.

12. Ferreira Júnior AJ, Zanetti GG, Couto PG, Bastos LAL, Silva SFD, Lima JRP, et al. Transition points of heart rate during a progressive maximal intermittent field test in young soccer players. J Exerc Physio Online. 2012;15(3):81-8

13. Lima JRP, Kiss MAPD. Limiar de variabilidade da frequência cardíaca. Rev Bras Ativ Fis Saúde. 1999;4(1):29-38

14. Nakamura FY, Aguiar CA, Fronchetti L, Aguiar AF, Lima JRP. Alteração do limiar de variabilidade da frequência cardíaca após treinamento aeróbio de curto prazo. Motriz, Rio Claro. 2005;11(1):1-9.

15. Costa VP, Lima JRP, Oliveira FR. Identificação de limiares metabólicos em curvas de frequência cardíaca ajustadas. Rev Bras Educ Fis Esp. 2007;21(3):219-27.
16. Erdogan A, Cetin C, Karatosun H, Baydar ML. Non-invasive indices for the estimation of the anaerobic threshold of oarsmen. J Int Med Res. 2010;38(3):901-15.

17. Conconi F, Grazzi G, Casoni I, Guglielmini C, Borsetto C, Ballarin E, et al. The Conconi test: methodology after 12 years of application. Int J Sports Med. 1996;17(7):509-19.

18. De-Oliveira FR. Prediccion de los umbrales de lactato y ajustes de frecuencia cardiaca en el test de legerboucher [tese]. San Sebastian, Sapain: Universidad del País Vasco, Facultad de Filosofia, y Ciências de la Educación; 2004

19. Carminatti L.Validade de limiares anaeróbios derivados do teste incremental de corrida intermitente (tcar) como preditores do máximo steady-state de lactato em jogadores de futsal [dissertação]. Florianópolis, SC: Universidade do Estado de Santa Catarina. Centro de Educação Física, Fisioterapia e Desportos; 2006.

20. Couto PG, Rodrigues AP, Ferreira-Junior AJ, Silva SF, De-Oliveira FR. Pontos de transição da frequência cardíaca em teste progressivo máximo. Motriz, Rio Claro. 2013;19(2):261-8.

21. Conconi F, Borsetto C, Casoni J, Ferrari M. Noninvasive determination of the anaerobic threshold in cyclists. Medical and Scientific Aspects in Cycling, Hum Kin. 1988;79-91

22. Hofmann P, Pokan R, Preidler K, Leitner H, Szolar D, Eber B, et al. Relationship between heart rate threshold, lactate turn point and myocardial function. Int J Sports Med. 1994;15(5):232-7.

23. Vachon JA, Bassett DR Jr, Clarke S. Validity of the heart rate deflection point as a predictor of lactate threshold during running. J Appl Physiol (1985). 1999;87(1):452-9.

24. Frainer DES, Roza TH, Morastoni CC, Pazin J, De-Oliveira FR. Teste progressivo de marcha atlética em pista: efeitos do treinamento de curto prazo. In : Congresso Brasileiro de Metabolismo, Nutrição e Exercício. Congresso Brasileiro de Metabolismo, Nutrição e Exercício, Londrina, PR, 2006.

25. Kara M, Gökbel H, Bediz C, Ergene N, Uçok K, Uysal H. Determination of the heart rate deflection point by the Dmax method. J Sports Med Phys Fitness. 1996;36(1):31-4.

26. McArdle WD, Katch FI, Katch VL. Fisiologia do exercício: energia, nutrição e desempenho humano. $6^{a}$ ed. Rio de Janeiro: Guanabara; 2008.

27. Ignjatović A, Hofmann P, Radovanović D. Non-invasive determination of the anaerobic threshold based on the heart rate deflection point. Ser Physiol Educ Sport. 2008:6(1):1-10.

28. Pokan R, Hofmann P, Preidler K, Leitner H, Dusleag J, Eber B, et al. Correlation between inflection of heart rate/work performance curve and myocardial function in exhausting cycle ergometer exercise. Eur $\rfloor$ Appl Physiol Occup Physiol. 1993;67(5):385-8.

29. Pokan R, Hofmann P, Lehmann M, Leitner H, Eber B, Gasser R, et al. Heart rate deflection related to lactate performance curve and plasma catecholamine response during incremental cycle ergometer exercise. Eur J Appl Physiol Occup Physiol. 1995;70(2):175-9.

30. Hofmann P, Wonisch M, Pokan R, Schwaberger G, Smekal G, Duvillard SP. B1- Adenoceptor mediated origin of the heart rate performance curve deflection. Med Sci Sports Exerc. 2005;37(10):1704-9. 